\title{
Microstructure et potentiel de gonflement d'une argile du Sahara algérien
}

M. LAMARA

Département de génie civil Université Ammar Télidji BP $37 G$

03000 Laghouat (Algérie) Irm3a_nd@yahoo.fr

Z. DERRICHE

École nationale supérieure des travaux publics

1, rue Sidi Garidi, BP 32

Vieux Kouba

16051 Alger (Algérie) zohra_derriche@yahoo.com

E. ROMERO

Department Geotechnical Engineering and Geosciences

Universitat Politècnica de Catalunya

Building D2, Campus Nord c/J. Girona 1.3

08034 Barcelone (Espagne) Enrique.romero. morales@upc.edu

Durant les années 80, une raffinerie de pétrole et des bâtiments résidentiels divers, construits dans la région d'In-Aménas (au sud-est de l'Algérie), ont subi des dommages sévères dus au gonflement du sol. Quelques années seulement après sa réception, la raffinerie a dû être fermée et les constructions touchées sont tombées en ruine et ont dû être déblayées. Des études réalisées sur le sol de la région ont montré qu'il s'agit d'une argile de type kaolinite contenant des interstratifiés : illitevermiculite. Ces argiles présentent habituellement un potentiel de gonflement relativement faible. Dans le but de comprendre pourquoi les argiles d'In-Aménas ont développé un gonflement aussi dévastateur, les résultats d'une campagne d'essais de gonflement ont été analysés. Des investigations expérimentales complémentaires portant sur la microstructure et les propriétés de rétention d'eau du sol ont été effectuées. A la lumière de ces derniers résultats, l'importance de la microstructure du sol sur son comportement au gonflement est mise en évidence.

Mots-clés : dessiccation, double porosité, microstructure, argile gonflante, rétention d'eau, kaolinite.

\section{Microstructure and swell behaviour of a clay from the Algerian Sahara}

During the eighties an oil refinery plant with its various constructions, built in the area of In-Aménas (South East of Algeria) in the Algerian Sahara, suffered severe damage as a result of ground heaving. Only a few years after construction completion, the refinery had to be closed. Its buildings were completely destroyed and had to be cleared. Studies carried out on the soils of the area show that the nature of the swelling clay is kaolinite with presence of interstratified illite-vermiculite. These clay minerals are usually associated with a relatively low swell potential. In order to understand why the In-Aménas clays have developed such a devastating swell behaviour, the results of a programme of various swell tests are thoroughly examined. Complementary experimental investigations related to the microstructure of the clay and to its water retention properties are carried out. In the light of the results obtained an analysis is presented to show the importance of the microstructural organization of the soil on its swell behaviour.

Key words: desiccation, double porosity, microstructure, swelling clay, water retention, kaolinite. 


\section{Introduction}

La présence d'argiles gonflantes cause de sérieux problèmes dans les régions arides. Le sol s'y trouve dans un état de dessiccation tellement poussé que l'apport d'une infime quantité d'eau peut provoquer des gonflements importants qui se traduisent par des désordres préjudiciables sur les constructions.

L'Algérie, comme de nombreux pays, souffre du problème du gonflement des sols. Compte tenu de l'importance et de la gravité des désordres, dus au gonflement, enregistrés dans plusieurs régions du Sud du pays, le gonflement des sols constitue un handicap majeur pour le développement de ces régions. Cela est d'autant plus vrai que ces régions, encore largement inhabitées, renferment l'essentiel des ressources pétrolières du pays.

L'expérience des dégâts dus au gonflement des sols vécue à In-Aménas est particulièrement singulière et n'en finit pas de susciter l'intérêt des chercheurs. Dans cette région du Sud-Est du pays, pour la plupart des ouvrages touchés, les désordres dus au gonflement débutent dès la réception et évoluent d'une manière anormalement rapide, tellement rapide que seulement quelques années suffisent pour mener les constructions à une ruine totale. L'exemple le plus spectaculaire enregistré dans la région est sans doute celui de la très grande installation de raffinage qui a été sévèrement éprouvée, usine et bâtiments annexes, par le gonflement des sols et a dû être abandonnée après quelque 5 à 6 années d'exploitation, interrompue par plusieurs campagnes de confortement. Les bâtiments annexes de la raffinerie, très endommagés, ont été déblayés. L'usine a été démantelée et le projet de son transfert à un autre endroit est actuellement encore à l'étude. Il est à noter qu'à In-Aménas, beaucoup d'infrastructures de base, aéroport et routes, sont maintenues en exploitation au prix d'onéreuses opérations de réparation. Une analyse approfondie du comportement des ouvrages de la raffinerie a été présentée dans Derriche et al. (1999).

Les sols fortement expansifs sont habituellement composés de minéraux de type smectite. Toutefois, sous certaines conditions, on a pu montrer qu'une argile kaolinitique associée à une faible proportion d'interstratifiés « illite-vermiculite », i.e. l'argile d'In-Aménas, pouvait exhiber un comportement au gonflement aussi important que celui des smectites et représenter un réel danger pour les constructions.

Pour ce faire, on a utilisé les résultats d'un programme d'essais de gonflement réalisés en conditions œdométriques. On a complété la caractérisation hydraulique de l'argile par la mesure des caractéristiques de rétention d'eau, obtenues par la méthode des psychromètres, en conditions non chargées. Pour révéler l'organisation microstructurale du matériau, à même d'expliquer l'importance du gonflement observé pour les argiles d'In-Aménas, on a analysé le réseau poral du sol à l'état naturel par porosimétrie à intrusion de mercure (MIP) sur des échantillons lyophilisés.

On a interprété les résultats obtenus pour proposer une explication possible au potentiel de gonflement particulièrement élevé développé par les sols de la région d'In-Aménas.

\section{Situation}

La région d'In-Aménas est située à 1600 kilomètres au sud-est d'Alger. Elle est repérée par les latitude 28-05 Nord et longitude 09-63 Est. La région se trouve à une altitude de $521 \mathrm{~m}$ et se caractérise par une morphologie plutôt plane avec absence quasi-totale de drainages naturels superficiels.

Le climat de la région est de type saharien avec une longue saison estivale sèche et une très courte saison hivernale douce. La valeur des précipitations est particulièrement faible $(29 \mathrm{~mm} / \mathrm{an})$. Les vents sont froids et secs en hiver et deviennent chauds et desséchants en été (sirocco). Le degré hygrométrique est très faible tout au long de l'année ; il varie de $20 \%$ en été à $50 \%$ en hiver. Ces valeurs de l'humidité relative correspondent à des succions totales de 95 à $210 \mathrm{MPa}$, en vertu de la loi psychrométrique considérée à $20^{\circ} \mathrm{C}$.

3

\section{Caractéristiques géotechniques - minéralogie}

\section{1}

\section{Géologie locale}

Les données géologiques locales ont montré que la formation principale rencontrée dans la région appartient à la série des argiles triasiques du Zarzataine ; région qui renferme les plus importants gisements pétroliers et gaziers du Sahara. Des sondages pétroliers ont montré que, par endroits, cette formation d'argile a une épaisseur supérieure à $180 \mathrm{~m}$ et surmonte une formation carbonifère très épaisse.

Les multiples études réalisées dans la région ont révélé la présence d'un sol composé essentiellement d'argile rouge, bariolée par endroits de mauve, de jaune, de vert et de bleu. Cette couche est très compacte et est couverte en surface par une couche d'altération de quelques centimètres. Dans sa partie supérieure, le sol est constitué d'un matériau limonoargileux, plus ou moins dépourvu de fraction sableuse et qui se présente en petits blocs très compacts séparés par de nombreuses fractures. Plus en profondeur, le sol est une argile limono-sableuse visiblement moins compacte que l'argile qui la surmonte.

Eu égard au caractère lithoïde particulièrement friable de l'argile, les résultats présentés dans cette étude ont concerné des échantillons manuellement taillés aux dimensions voulues (avec couteau et papier abrasif) dans des blocs récupérés à une profondeur d'environ $4 \mathrm{~m}$, dans des tranchées exploratrices et puits de reconnaissance réalisés par l'entreprise SONATRACH.

\section{2}

\section{Composition chimique}

Les analyses chimiques réalisées sur l'argile ont mis en évidence une grande proportion de silicates (>50\%) comparée à celle des aluminates $(<20 \%)$. Ces proportions donnent au sol un rapport silice-alumine 
et silice-sesquioxydes important comparable à celui des argiles de type smectique. Ce rapport est manifestement influencé par la présence de silice cristallisée. Les résultats indiquent la présence d'un pourcentage non négligeable de minéraux argileux de type $2: 1$ ( 2 motifs tétraédriques ; 1 motif octaédrique).

La teneur de $\mathrm{K}_{2} \mathrm{O}$ confirme la présence d'illite dans les proportions de 20 à 28 \% (Derriche et al., 1999). La proportion d'oxydes ferriques (5 à 8,4\%) indique le caractère ferrugineux, par ailleurs apparent dans la couleur rougeâtre dominante de l'argile.

La mesure du $\mathrm{pH}$ a donné des valeurs comprises entre 7,2 et 7,8 démontrant le caractère alcalin du sol. La présence des cations échangeables basiques $(\mathrm{Ca}$, $\mathrm{Mg}, \mathrm{Na}$ et K) a été évaluée à $22,35 \mathrm{meq} / 100 \mathrm{~g}$, avec plus de la moitié assurée par le calcium (12,8 meq/100 g).

\section{3}

\section{Caractéristiques physiques}

A l'état naturel, l'argile présente la consistance d'une roche dure et friable. Sa densité est très forte (2 à 2,1). La composition granulométrique montre que le sol contient une large proportion (50 à $80 \%$ ) de particules fines $(<2 \mu \mathrm{m})$ (Fig. 1a). La limite de liquidité varie entre 34 et $80 \%$ et l'indice de plasticité entre 23 et $45 \%$ (Fig. 1b). La limite de retrait varie entre 10 et $14 \%$, et la teneur en eau naturelle est très faible et souvent comprise entre 1,7 et $4 \%$. Ces valeurs sont en conformité avec la forte compacité du sol et sont la conséquence de la surconsolidation poussée, induite par la forte dessiccation des dépôts d'argile de la région.

Les analyses par diffraction aux rayons $\mathrm{X}$ de trois échantillons ont révélé que ces argiles sont à prédominance de kaolinite avec la présence, en moindre importance, d'interstratifiés (c illite-vermiculite ») et de silice cristallisée. Ces minéraux argileux sont normalement associés à un potentiel de gonflement faible.

On donne sur la figure $1 \mathrm{~b}$ une représentation de la plasticité de l'argile d'In-Aménas en même temps que celle de l'argile de Boom (Romero, 1999), qui est une argile modérément gonflante de type kaolinitique-illitique et très similaire en termes de minéralogie à l'argile d’In-Aménas. Les résultats de l'argile de Boom utilisés

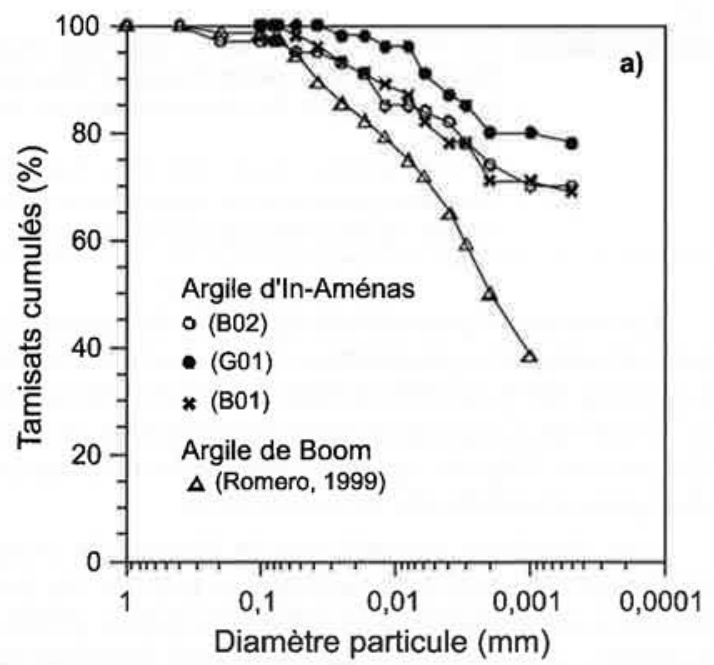

dans cette étude concernent des échantillons compactés statiquement du côté sec de la courbe de compactage à une densité sèche de 1,67 (Romero, 1999).

On remarque que, pour les deux sols, les points représentatifs de la plasticité se situent le long de la ligne A de l'abaque de Casagrande. Selon Sridharan (2005), très souvent cette caractéristique, commune aux sols kaolinitiques et montmorillonitiques, ne permet pas de distinguer la présence de la première classe d'argile de la seconde à partir de cette classification conventionnelle de la plasticité.

A première vue, il semble évident que les deux argiles présentent des plasticités comparables.

\section{4}

\section{Méthodes d'essais}

\section{1 \\ Porosimétrie à intrusion de mercure}

L'essai de porosimétrie à intrusion de mercure (MIP) a été utilisé pour fournir des informations sur l'organisation du réseau poral du sol d'In-Aménas, considérée à l'échelle de l'agrégat individuel ainsi qu'à celui de l'agencement des agrégats entre eux. Ces informations pouvaient mettre en évidence les caractéristiques microstructurales à même d'expliquer l'importance du potentiel de gonflement de l'argile d'In Aménas.

Les essais MIP ont été réalisés suivant la procédure ASTM D4404, avec l'appareil Micromeritics-AutoPore IV, capable d'atteindre une pression d'intrusion de $220 \mathrm{MPa}$. La dimension des pores, qui peut être mesurée par cet appareil, varie de $6 \mathrm{~nm}$ à $400 \mu \mathrm{m}$. Bien que le matériau testé présente une humidité très faible (très inférieure à la limite de retrait), un processus de lyophilisation a été employé pour sécher les échantillons en veillant à la conservation de leur organisation porale (Delage et al., 1982). Deux types de porosité peuvent être caractérisés par un cycle injection-extrusion de mercure dans le sol (Roméro, 1999). Lors de l'opération d'injection, le mercure remplit tout le volume des pores interconnectés qui sont accessibles dans la porosité du sol. En revanche, lors du chemin inverse, la libéra-

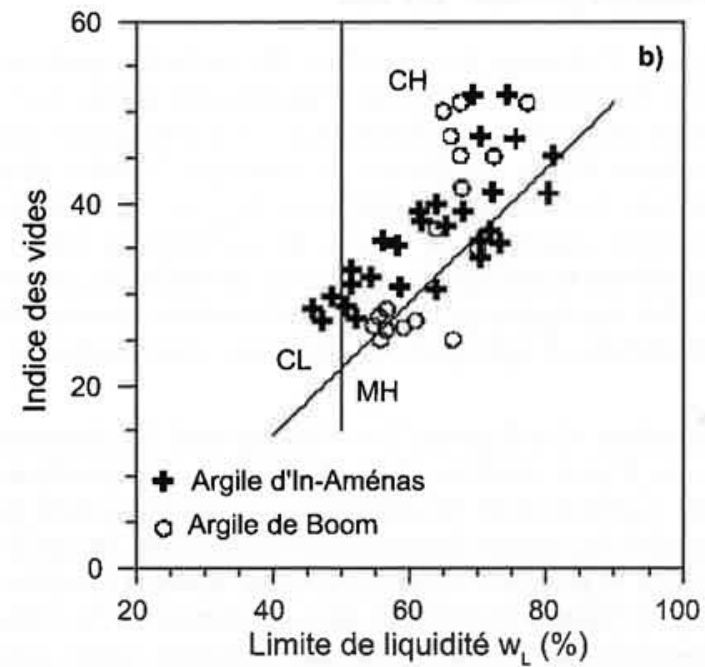

a) Courbes granulométriques de trois échantillons de l'argile d'In-Aménas et d'un échantillon de l'argile de Boom. b) Représentation de la plasticité dans l'abaque de Casagrande.

a) Particle size distribution of three samples of In-Aménas clay and that of Boom Clay. b) Plasticity chart. 
tion totale de la pression ne peut expulser que seulement une partie du mercure présent dans les pores. La différence entre les effets de la phase d'injection sous pression et ceux de la libération de la pression met en évidence la porosité piégée du sol. Selon Delage et Pellerin (1984), la porosité piégée représente la porosité inter-agrégats (macro-porosité), tandis que la porosité libre correspond au volume des vides intra-agrégats.

\section{2 .}

\section{Courbe caractéristique de retention d'eau}

Des psychromètres à transistors (SMI, Woodburn et al., 1993), avec une plage de mesure étendue, ont été utilisés pour l'obtention de la courbe de rétention d'eau (exprimée en succion totale pouvant varier de 0,5 à $80 \mathrm{MPa}$ ). Le voltage produit en sortie par le transistor peut être relié à l'humidité relative (ou à la succion totale via la loi psychrométrique) à travers un étalonnage approprié (Mata et al., 2002). Les mesures ont été réalisées sur deux spécimens prélevés dans le même échantillon. Les spécimens ont été mouillés par étapes, stockés pendant une journée pour équilibrage de l'humidité. Les spécimens ont été ensuite pesés et l'humidité relative de l'air les entourant mesurée. A la fin de la procédure de mouillage en multi-étapes, les spécimens ont été pesés, séchés à l'étuve pour déterminer leur teneur en eau.

\section{3}

\section{Caractéristiques de gonflement}

Les résultats des essais de gonflement présentés dans cet article ont été empruntés à Tas (1992) qui a réalisé une étude approfondie du gonflement à l'œdomètre des sols d'In-Aménas.

\section{5}

\section{Résultats et interprétation}

\section{1}

\section{Organisation porale du sol}

La figure 2 donne les courbes du volume poreux injecté en fonction du rayon d'accès du pore, correspondant aux phases d'injection et d'extrusion du mercure dans le sol. La figure 3a montre l'indice des vides cumulé injecté par le mercure $\left(\mathrm{e}_{\mathrm{nw}}\right.$ le volume de mercure entré dans le sol rapporté au volume solide de l'échantillon testé) en fonction de la taille du pore d'entrée des agrégats (à l'échelle microstructurale) et des agglomérats d'agrégats (à l'échelle macrostructurale).

Les courbes des figures 2 et 3 indiquent l'existence dans le sol d'une distribution bimodale discontinue des pores. Celle-ci met en évidence à une extrémité la présence de très larges pores (inter-agrégats), de taille supérieure à $10 \mu \mathrm{m}$ avec une taille dominante d'environ $360 \mu \mathrm{m}$, et à l'autre extrémité des micropores de très faible diamètre, inférieur à $100 \mathrm{~nm}(0,1 \mu \mathrm{m})$, avec une taille dominante de $10 \mathrm{~nm}(100 \AA)$. Les pores de diamètres intermédiaires, ceux compris entre 0,1 et $10 \mu \mathrm{m}$, ne semblent pas exister dans l'argile d'In-Aménas.

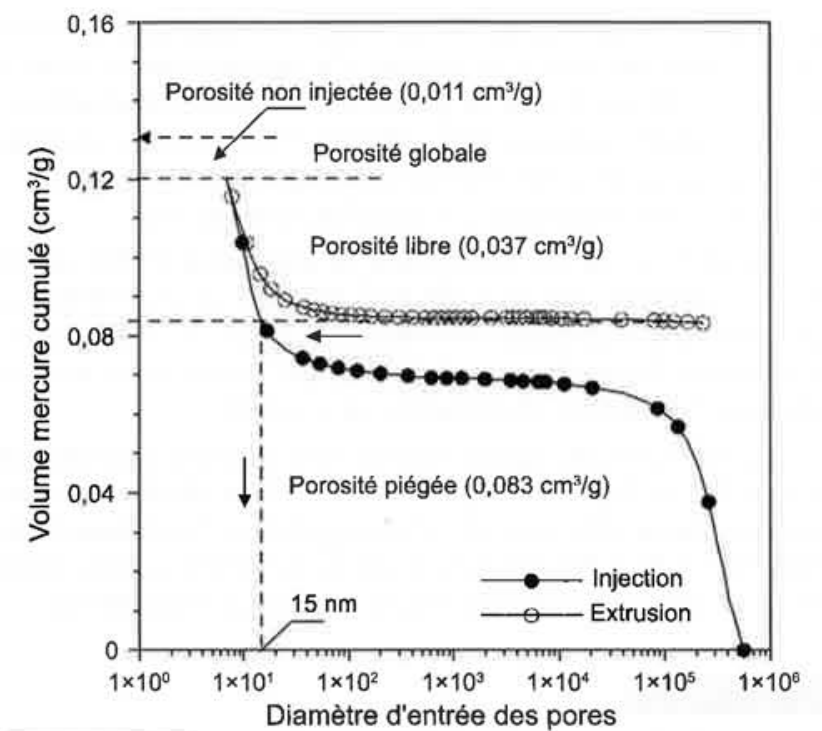

nc.2 Courbes porosimétriques d'injection extrusion de mercure.

Mercury intrusion - extrusion porosimetry curves.

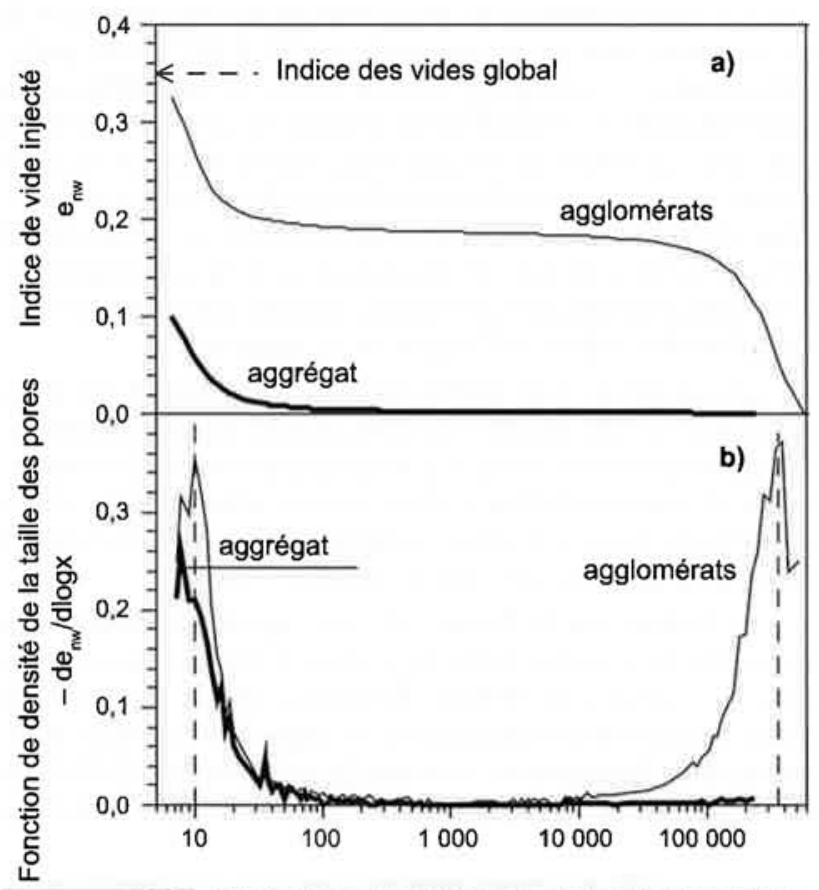

F16.3 a) Indice des vides cumulé injecté en fonction de la taille d'entrée des pores. b) Fonctions de densité de la taille des pores.

a) Cumulative void ratio as a function of the entrance pore size for aggregation and packing scales. b) Pore size density functions.

Les courbes porosimétriques de la figure 2 permettent d'évaluer la proportion de la porosité libre du sol à près de $30 \%\left(0,037 \mathrm{~cm}^{3} / \mathrm{g}\right)$ de la porosité totale du sol $\left(0,12 \mathrm{~cm}^{3} / \mathrm{g}\right)$. Ce pourcentage correspond, sur la courbe d'injection (Fig. 4), approximativement à des pores de diamètre d'entrée de $15 \mathrm{~nm}(150 \AA)$.

Les résultats donnés sur la figure $3 \mathrm{~b}$ complètent l'information sur l'organisation porale du sol par la fonction de densité de la taille des pores (PSD), définie comme - $\mathrm{de}_{\mathrm{nw}} / \mathrm{d} \log \mathrm{x}$, pour les deux échelles de porosité (agrégats et agglomérats). Ces résultats indiquent que la courbe PSD des agrégats affiche un seul mode correspondant à une taille d'entrée des pores extrê- 


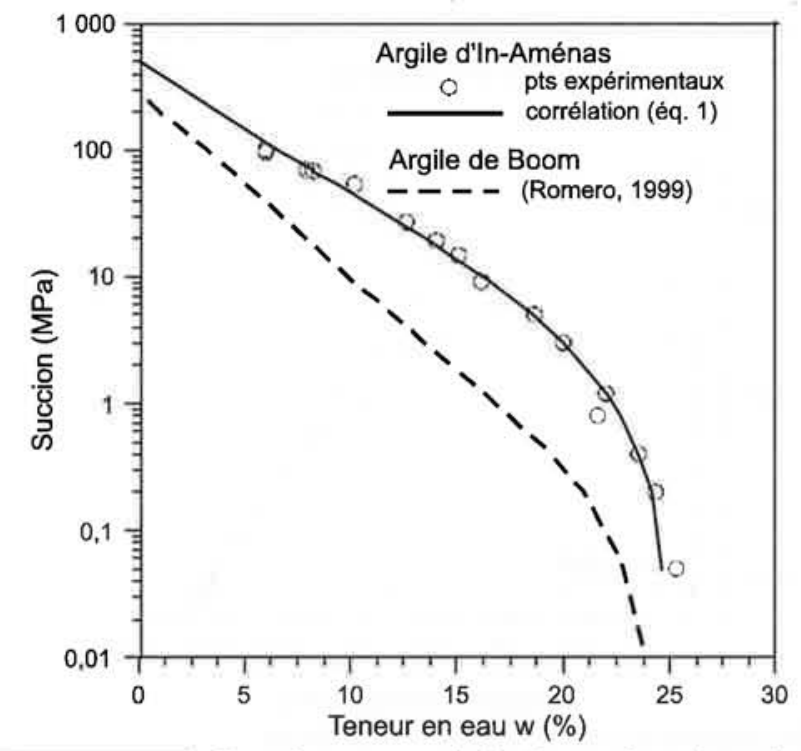

Fic.4 Courbe caractéristique de rétention d'eau de l'argile d'In-Aménas (chemin d'humidification en conditions non chargées) et celle de l'argile de Boom présentée à des fins de comparaison.

Water retention properties of In-Aménas clay (wetting path under unstressed conditions). Water retention curve of the Boom's clay plotted for comparison.

mement petite, de l'ordre de $10 \mathrm{~nm}$, valeur qui est en accord avec la densité très élevée du matériau.

Cette valeur est aussi proche de la taille d'entrée de pore minimum détectable par l'appareil utilisé. Le recours à un porosimètre d'adsorption de nitrogène permettrait de confirmer ce mode.

En revanche, et comme indiqué plus haut, l'échelle des agglomérats est clairement bi-modale :

- d'une part, les modes à l'échelle des agglomérats correspondent à la même taille des pores intra-agrégats (les pores à l'intérieur des agrégats) $10 \mathrm{~nm}$;

- d'autre part, la porosité des inter-agglomérats est représentée par la taille particulièrement importante des pores de $360 \mu \mathrm{m}$. Ce résultat paraît normal compte tenu de l'état d'extrême dessiccation du sol.

L'indice des vides maximal injecté au niveau des agrégats est de 0,10 , ce qui correspond à une densité sèche des agrégats, très importante (proche de $2,45)$. Il est vrai qu'à cause de la limitation de la capacité de l'appareillage à distinguer les pores très petits des agrégats, il y a évidemment un pourcentage de porosité non injectée. On évalue cet écart, par rapport à l'indice des vides global estimé $(\mathrm{e}=0,355)$, à $\Delta \mathrm{e}=0,036$ (Fig. 2). A cause de cet écart, la densité sèche des agrégats ne devrait être que légèrement inférieure à la valeur indiquée précédemment.

\section{2}

\section{Courbe de rétention d'eau}

Les points expérimentaux obtenus sont présentés sur la figure 4, avec une courbe de corrélation utilisant une forme modifiée de l'équation de van Genuchten (1980), dans laquelle la teneur en eau w est définie comme une fonction de la succion (Romero et Vaunat, 2000).
Dans l'équation (1), les paramètres $n$ et $m$ sont les mêmes que ceux de l'expression originale de van Genuchten, $w_{\text {sat }}$ représente la teneur en eau du sol en conditions saturées, et $\mathrm{S}_{r}$ est le degré de saturation du sol. La corrélation donnée dans l'équation (1) force la courbe de rétention à être linéaire dans une échelle semi-logarithmique dans le domaine des succions élevées. Le paramètre a représente l'intersection avec l'axe y correspondant à une teneur en eau nulle dans cette partie linéaire. Un algorithme utilisant la méthode des moindres carrés est utilisé pour déterminer les paramètres du modèle $(n, m$, et $\alpha$ ), supposant $a=500 \mathrm{MPa}$ et $\mathrm{w}_{\text {sat }}=25,3 \%$. Les paramètres trouvés sont $\mathrm{n}=1, \mathrm{~m}$ $=0,64$ et $\alpha=0,09 \mathrm{MPa}^{-1}$.

$$
\frac{\mathrm{W}}{\mathrm{W}_{\mathrm{sat}}}=\mathrm{S}_{\mathrm{r}}=\mathrm{C}(\mathrm{s})\left[\frac{1}{1+(\alpha \mathrm{s})^{\mathrm{n}}}\right]^{\mathrm{m}}, \mathrm{C}(\mathrm{s})=1-\frac{\ln \left[1+\frac{\mathrm{s}}{\mathrm{a}}\right]}{\ln (2)}
$$

La figure 4 présente, à des fins de comparaison, la courbe de rétention de l'argile de Boom, une argile kaolinitique-illitique (Romero, 1999) très similaire à l'argile d'In-Aménas. Cette argile modérément gonflante présente une teneur en eau à la saturation et une limite de liquidité similaires à celles de l'argile d'In-Aménas, bien que la proportion de fines $(<2 \mu \mathrm{m})$ de l'argile de Boom soit inférieure $(50 \%)$. Cependant, pour la même humidité relative (ou succion totale) l'argile d'In-Aménas est capable de retenir plus d'eau que l'argile de Boom. Cette hygroscopicité élevée du sol d'In-Aménas se traduit par une capacité de variation de volume en réponse à l'augmentation de l'humidité relative.

\section{3 \\ Gonflement}

La figure 5 a montre la cinétique de gonflement sous conditions œdométriques de deux échantillons d'argile, saturés et autorisés à gonfler librement sous le poids du piston $(<2 \mathrm{kPa})$. La déformation de gonflement développée par l'échantillon (couche supérieure) est d'environ $20 \%$ après une durée de 11 jours. Des déformations de gonflement plus importantes (> $30 \%$ ) ont été enregistrées sur d'autres échantillons (Tas, 1992). Sur les courbes de la figure $5 a$, les phases de gonflement primaire et secondaire sont clairement identifiées. Le gonflement secondaire commence à partir d'une journée et semble se stabiliser au-delà d'une période de 14 jours (20 $000 \mathrm{~min}$ ). Il est habituellement associé avec l'hydratation progressive des agrégats de forte densité.

Au mouillage, l'eau remplit les macro-pores du sol et s'écoule à travers, conférant alors une grande perméabilité au sol. Les forces de capillarité inter-granulaires, qui maintiennent l'assemblage des agrégats naturels stables, sont rapidement modifiées, ce qui provoque immédiatement un gonflement primaire. Prend place par la suite au niveau de la microstructure (à l'intérieur des agrégats) et des agrégats potentiellement expansifs un gonflement secondaire qui s'alimente de l'eau disponible dans les macro-pores du sol.

Le transfert de l'eau entre les agrégats expansifs et la macro-porosité ouverte continue jusqu'à ce qu'un équilibre soit atteint. Cette explication inclut implicitement que coexistent à l'intérieur de l'échantillon deux différents potentiels de l'eau. L'eau remplissant les macro-pores possède un macro-potentiel qui n'est pas 

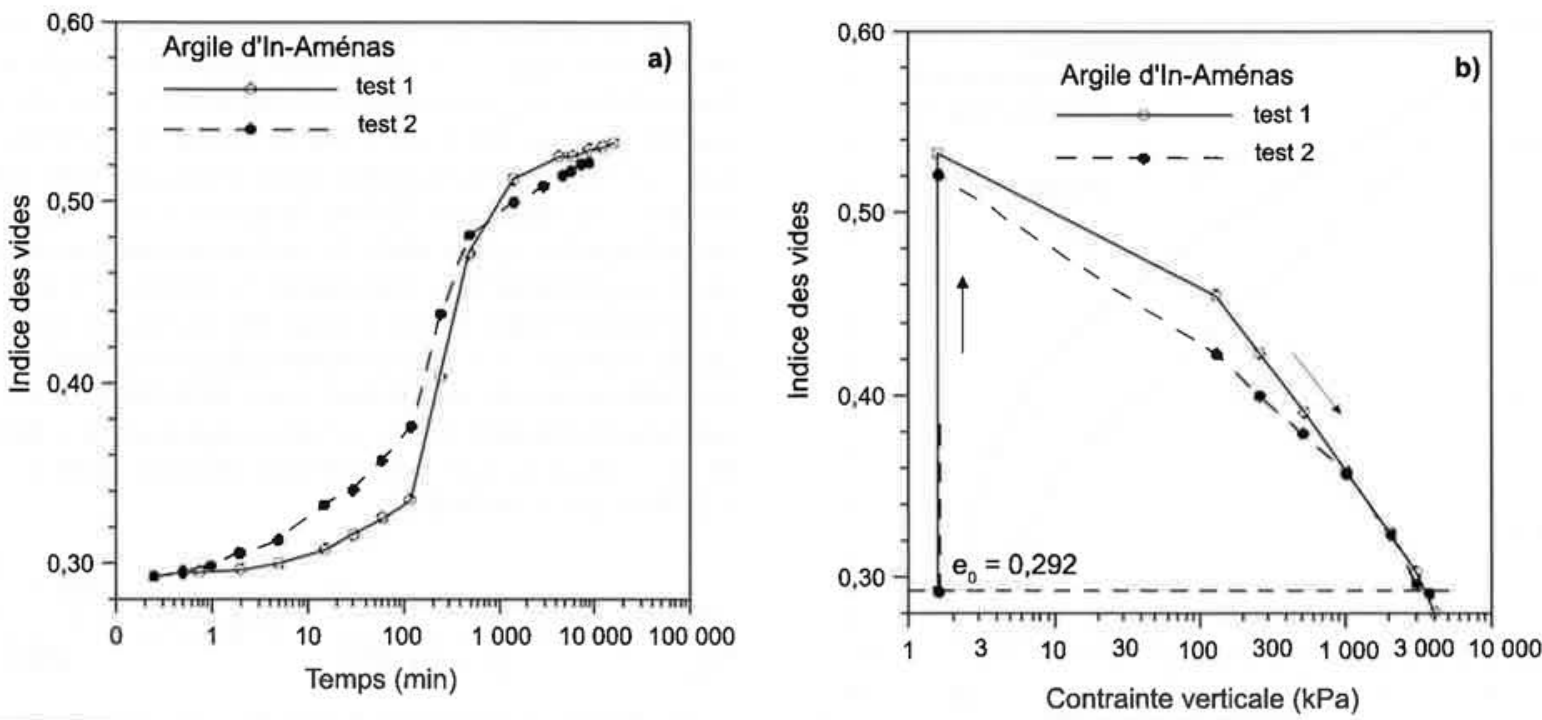

56.5. Gonflement libre de deux échantillons de la couche supérieure de l'argile d'In-Aménas.

a) Cinétique de gonflement. b) Pression de gonflement obtenue par chargement après gonflement libre. Swell potential of two samples from top layer of In-Aménas clay. a) Time evolution of swell deformation. b) Swell pressure obtained by loading following free swell.

nécessairement en équilibre avec le potentiel de l'eau à l'intérieur des agrégats. L'organisation du réseau des pores, pour les deux niveaux macro et microstructuraux de l'argile d'In-Aménas, est analysée dans les sections précédentes.

La figure $5 \mathrm{~b}$ présente les résultats de deux essais de chargement après gonflement libre. Les échantillons sont ramenés à leur indice des vides initial par l'application d'une pression verticale supérieure à $3000 \mathrm{kPa}$. Cette valeur peut représenter une limite supérieure pour la pression de gonflement réelle du sol. A noter par ailleurs que des pressions de gonflement de l'ordre de 6000 à $8000 \mathrm{kPa}$ ont été mesurées pour certains échantillons. Il est bien connu que la pression de gonflement, déterminée par chargement après gonflement libre, est habituellement plus importante comparée à celle obtenue par gonflement sous charges constantes et celle donnée par l'essai à volume constant (Gens et Alonso, 1992). Il ressort néanmoins de ces résultats que l'argile d'In-Aménas est potentiellement gonflante. Elle peut exhiber des déformations de gonflement importantes (>30\%) et développer des pressions de gonflement considérables ( 3 à $8 \mathrm{MPa}$ ).

On donne sur la figure $6 \mathrm{a}$, les courbes de chargement après gonflement d'échantillons saturés et autorisés à gonfler sous des charges constantes de 500, 1000 et $2000 \mathrm{kPa}$, Les échantillons saturés sous chargement montrent un gonflement réduit. Après inondation, l'échantillon testé, sous la charge de $2000 \mathrm{kPa}$, a d'abord amorcé un très faible tassement avant
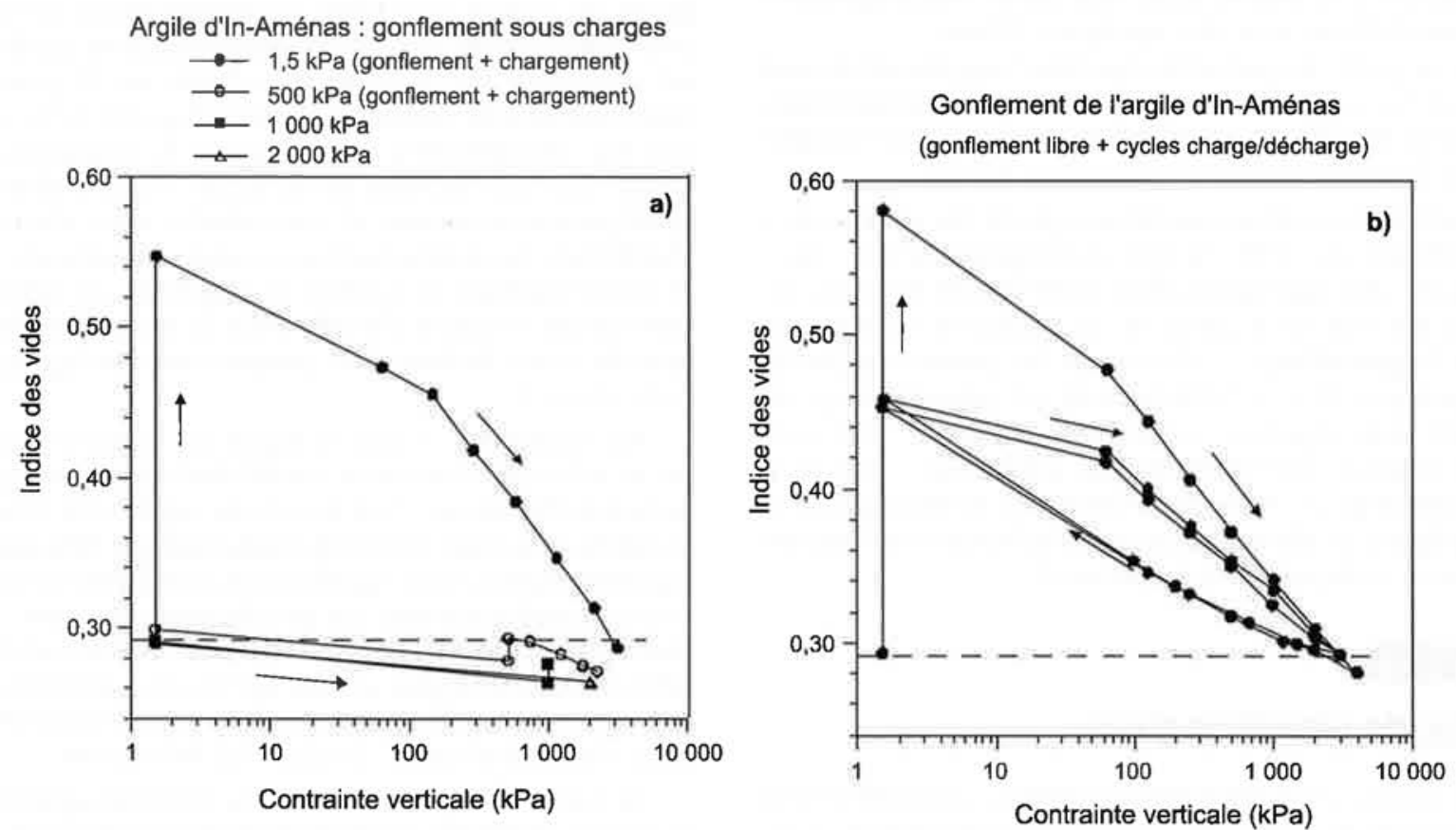

Fi6.6 a) Comparaison gonflement libre - gonflement sous charges.

b) Cycles de chargement - déchargement après gonflement libre (Tas, 1992).

a) Comparison between free swell and swell under constant load.

b) Cyclic loading and unloading after free swelling (Tas, 1992). 
d'entamer un gonflement. On suppose que, durant le mouillage progressif du sol, les agrégats naturels, initialement très denses, subissent un relâchement accompagné d'une réduction de résistance et de rigidité, et fusionnent ensemble en remplissant les vides inter-agrégats. Sous contrainte élevée, le phénomène est équivalent à l'échelle macroscopique à ce que le matériau subit un effondrement dû au mouillage. Cependant, le comportement expansif reste dominant et un gonflement net est toujours mesuré.

La figure $6 \mathrm{~b}$ représente les courbes décrivant des cycles de chargement-déchargement après un chemin de gonflement libre sous le poids du piston. Ces courbes montrent que le comportement du sol est approximativement réversible après seulement le premier cycle de chargement. La déformation volumique réversible représente environ $60 \%$ de la déformation de gonflement initial enregistrée pendant le gonflement libre. Le gonflement observé lors du mouillage du sol dépend de la microstructure du sol et de la contrainte mécanique subie. Il semble qu'au cours du mouillage, sous contrainte mécanique importante, l'effondrement détruit la microporosité initiale du sol.

Si on admet que la structuration initiale du sol est détruite par le chargement après le gonflement libre, la déformation réversible représenterait cette partie du gonflement qu'on peut qualifier d'intrinsèque au type du sol. Elle exprimerait l'affinité du sol à l'eau qui dépend de la minéralogie du sol i.e. de la surface spécifique, la CEC etc., et qui est en principe indépendante de l'organisation microstructurale des agrégats.

On remarque par ailleurs que le changement de la compressibilité du sol dans le domaine surconsolidé est particulièrement significatif lorsqu'on compare la compressibilité du matériau avant inondation (Fig. 6a) et sa compressibilité après gonflement libre et premier chemin de chargement (Fig. 6b). La première est contrôlée par la résistance au cisaillement aux alentours des points de contact des agrégats. La deuxième est gouvernée principalement par les phénomènes d'adsorption des doubles couches d'eau. Avec l'imbibition, la modification de la structure du sol est le résultat de la perte de résistance des agrégats et des agglomérats que les agrégats forment dans le sol.

Ainsi, la valeur très élevée de la densité sèche des agrégats peut donner une explication suffisante au potentiel de gonflement très important du sol, qui a priori n'est pas conforme à l'activité des minéraux argileux présents dans le sol.

En effet, d'après les résultats porosimétriques obtenus, l'argile d'In-Aménas peut être considérée comme présentant une structure franchement ouverte puisqu'elle possède des vides de grandes tailles occupant plus de $70 \%$ de la porosité totale du sol.

Lors du chemin de dessiccation, pour des humidités inférieures à la limite de retrait de l'argile (10 et $14 \%$ ), alors que le volume global du sol ne varie plus, les agrégats d'argile peuvent continuer à se retirer à l'intérieur du sol sous l'effet des succions élevées (telles que celles s'exerçant sur les sols d'In-Aménas, i.e. $100 \mathrm{MPa}$ en hiver et $200 \mathrm{MPa}$ en été), enregistrant des densités importantes et ménageant à l'intérieur du sol des pores inter-agrégats de plus en plus importants.

La dessiccation suscite l'agglomération des particules et crée souvent des liaisons chimiques des minéraux argileux avec les cations présents favorisées par le milieu alcalin du sol (Tessier et Pedro, 1976). A ce titre, la liaison fer-argile et le rôle du fer dans l'organisation des sols kaolinitiques ont été étudiés depuis longtemps par les pédologues. On attribue souvent au fer un rôle de ciment lié à la précipitation d'hydrates ferriques et à leur possibilité de floculation mutuelle avec l'argile (Chauvel et al., 1976). Il n'est pas impossible que la grande densité des agrégats soit en partie due à des réactions chimiques entre la kaolinite et le fer présent dans le sol.

Lors d'un chemin de mouillage, il est attendu que la taille des micropores à l'intérieur des agrégats augmente induisant une expansion importante de la microstructure. La possibilité du développement de la porosité entre les agrégats, conséquence de l'ex pansion de la microstructure sous faible contrainte, va aussi contribuer à l'augmentation de la capacité de gonflement du matériau.

\section{6 \\ Conclusion}

Le gonflement des sols représente un sérieux handicap pour le développement des régions arides du Sahara Algérien. Afin de donner la mesure de cet aléa, le cas spectaculaire de désordres causés par le gonflement du sol sous une installation de raffinage de pétrole dans la région de In-Aménas a été décrit.

Pour expliquer le gonflement singulier développé par les sols de cette région, jugé a priori non conforme aux minéraux argileux présents, des résultats d'essais de gonflement réalisés sous conditions œedométriques suivant divers chemins de contraintes mécaniques ont été complétés par des investigations portant sur la microstructure et les propriétés de rétention d'eau du sol.

L'argile d'In-Aménas est constituée de kaolinite avec présence d'interstratifiés illite-vermiculite. Elle présente une activité modérée mais se caractérise par une capacité de gonflement particulièrement importante. Cette capacité s'exprime par un potentiel de gonflement libre variant de $20 \%$ à plus de $30 \%$ et une pression de gonflement de l'ordre de 3 à $8 \mathrm{MPa}$ obtenue par chargement après gonflement libre sous le poids du piston

Les aspects microstructuraux de l'argile ont été analysés par porosimétrie à intrusion de mercure. Ces essais ont permis de montrer que l'argile d'In-Aménas présente une structure franchement ouverte formée d'agrégats très denses $(2,45)$ avec une taille de pore dominante de $10 \mathrm{~nm}$, et de vides inter-agrégats de très grandes tailles (de l'ordre de $360 \mu \mathrm{m}$ ) occupant $70 \%$ de la porosité totale du sol.

Cette microstructuration de l'argile est le résultat d'une forte dessiccation du sol à laquelle auraient contribué des réactions chimiques des minéraux argileux avec les cations présents donnant lieu à des oxydes basiques, ayant participé à la cimentation des agrégats argileux.

Le gonflement du sol dépend de la minéralogie de l'argile présente (gonflement intrinsèque), de la microstructure et de la contrainte mécanique subie par le sol. Les argiles de la région d'In-Aménas représentent un excellent exemple de l'importance des effets de la micro structure sur la capacité de gonflement affichée par le sol. 
Pour compléter la caractérisation hydraulique du sol, la courbe de rétention d'eau a été déterminée sous conditions non chargées. La structure ouverte de l'argile d'In-Aménas associée à son affinité intrinsèque à l'eau est à la base de sa grande capacité de rétention d'eau. Celle-ci a pu être vérifiée par comparaison avec celle d'une argile kaolinique-illitique, très similaire en termes de minéralogie et de plasticité à l'argile d'In Aménas, mais présentant manifestement une organisation microstructurale totalement différente. Si l'on admet que le gonflement sous faible contrainte préserve la structure initiale du sol, on peut affirmer que la structure ouverte de l'argile d'InAménas, conservée après gonflement, peut exercer un effet sur la perméabilité du sol. La vitesse de développement des désordres sur les constructions légères d'In-Aménas pourrait être due à une perméabilité importante du sol. Ainsi la microstructuration particulièrement ouverte de l'argile d'In-Aménas serait responsable non seulement de l'importante capacité de gonflement de l'argile mais vraisemblablement aussi de la vitesse à laquelle se développe ce gonflement. Des études complémentaires devraient confirmer ces hypothèses.

\section{Bibliographie}

Chauvel A., Pedro G., Tessier D. - Rôle du fer dans l'organisation des matériaux kaolinitiques : études expérimentales. Sciences du sol, n² 2, 1976, p. 101-113.

Delage P., Tessier D., Audiguier M.M. - Use of the cryoscan apparatus for observation of freeze-fractured planes of a sensitive Quebec clay in scanning electron microscopy. Canadian Geotechnical Journal, 19, 1982, p.111-114.

Delage P., Pellerin F.M. - Influence de la lyophilisation sur la structure d'une argile sensible du Québec. Clay Minerals, 19, 1984, p. 151-160.

Derriche Z., Iguechtal L., Tas M. - Comportement des ouvrages dans les argiles expansives d'In-Aménas. Revue française de géotechnique, 89, 1999, p. 55-65.

Gens A., Alonso, E.E. - A framework for the behaviour of unsaturated expansive clays. Canadian Geotechnical Journal, 29, 1992, p. 1013-1032.
Mata C., Romero E., Ledesma A. - Hydrochemical effects on water retention in bentonite-sand mixtures. Proceedings. $3^{\text {rd }}$ International Conference on unsaturated soils, Recife, Brazil, vol. 1, Jucá, de Campos \& Marinho (eds.), Swet \& Zeitlinger, Lisse, 2002, p. 283-288.

Romero E. - Characterization and thermo hydro-mechanical behaviour of unsaturated Boom clay : an experimental study. $\mathrm{PhD}$ Thesis, Universitat Politècnica de Catalunya, Spain, 1999.

Romero E., Vaunat J. - Retention curves of deformable clays. Proceedings international workshop on unsaturated soils : experimental evidence and theoretical approaches in unsaturated soils. A. Tarantino \& C. Mancuso (eds). A.A. Balkema, 2000, p. 10-12.

Sridharan A. - On swelling behaviour of clays. Proceedings international conference on problematic soils. GEOPROB, vol. 2, 2005, p. 499-516.
Tas M. - Influence des procédures expérimentales sur l'estimation en laboratoire des paramètres de gonflement des argiles expansives. Thèse de Magister, École nationale polytechnique d'Alger, 1992.

Tessier D., Pedro G. - Les modalités de l'organisation des particules dans les matériaux argileux : évolution des principales argiles $\mathrm{Ca}$ au cours du phénomène de retrait. Sciences du sol, $n^{\circ} 2,1976$, p. $85-100$.

van Genuchten M. Th. - A closed-form equation for predicting the hydraulic conductivity of unsaturated soils. Soil Science Society of America Journal, 44, 1980, p. 892-898.

Woodburn J.A., Hold. J., Peter, P. - The transistor psychrometer : a new instrument for measuring soil suction. Unsaturated Soils Geotechnical Special Publication $n^{\circ} 39$, Dallas, S.L. Houston \& W.K. Wray (eds). ASCE, 1993, p. 91-102. 